\title{
Oral Dexamethasone versus Oral Prednisolone in Acute Asthma: A New Randomized Controlled Trial and Updated Meta-analysis.
}

Source Citation: Paniagua N, Lopez R, Muñoz N, Tames M, Mojica E, Arana-Arri E, et al. Randomized trial of dexamethasone versus prednisone for children with acute asthma exacerbations. J Pediatr. 2017;191:190-6.

SECTION EDITOR: ABHIJEET SAHA

\section{SUMMARY}

This randomized, noninferiority trial included patients aged 1-14 years who presented to the emergency department (ED) with acute asthma. Primary objective was to compare the efficacy of two doses of dexamethasone $(0.6 \mathrm{mg} / \mathrm{kg} / \mathrm{dose}$, experimental treatment $)$ and a 5-day course of prednisolone/prednisone $(1.5 \mathrm{mg} /$ $\mathrm{kg} / \mathrm{d}$, followed by $1 \mathrm{mg} / \mathrm{kg} / \mathrm{d}$ on days $2-5$, conventional treatment). The primary outcome measures were the percentage of patients with asthma symptoms and quality of life at day 7. Secondary outcomes were unscheduled returns, admissions, adherence, and vomiting.

During the study period, 710 children who met the inclusion criteria were invited to participate and 590 agreed. Primary outcome data were available in 557 patients. At day 7, experimental and conventional groups did not show differences related to persistence of symptoms $(56.6 \%, 95 \%$ CI 50.6 to 62.6 vs $58.3 \%$, 95\% CI 52.3 to 64.2 , respectively), quality of life score (80.0 vs $77.7)$, admission rate (23.9\% vs $21.7 \%$ ), unscheduled ED return visits ( $4.6 \%$ vs $3.3 \%$ ), and vomiting (2.1\% vs $4.4 \%$ ). Adherence was greater in the dexamethasone group (99.3\% vs 96.0\%, $P<0.05$ ). Authors concluded that two doses of dexamethasone may be an effective alternative to a 5-day course of prednisone/prednisolone for asthma exacerbations, as measured by persistence of symptoms and quality of life at day 7 .

\section{Commentaries}

\section{Evidence-based Medicine Viewpoint}

Relevance: Administration of parenteral corticosteroids is a standard of care for acute asthma exacerbations in children and adults. This is reflected in most evidencebased guidelines, irrespective of whether initial management is started at home, primary health-care facilities or hospitals [1-4]. In fact, these guidelines recommend initiation of steroid therapy within the first hour of management in all except mild exacerbations. Oral administration has been shown to be as effective as intravenous or intramuscular administration. Thus, oral prednisone/prednisolone in the dose of $1-2 \mathrm{mg} / \mathrm{kg}$ per day has been recommended for a total of 5-7 days; although some studies have examined shorter courses and/or lower doses.

A limited number of studies also compared prednisone versus dexamethasone with the goal of evaluating whether the duration of therapy and/or number of doses could be reduced. There are four reasonably well-designed trials comparing oral prednisone versus oral dexamethasone in children [5-8]. A relatively recent Cochrane systematic review [9] reported that both medications had comparable efficacy in terms of hospital admission frequency (OR 1.08; 95\% CI 0.74, 1.58; 3 trials; 1007 participants), re-admission to hospital (OR 0.44 ; $95 \%$ CI $0.15,1.33 ; 3$ trials; 985 participants), new exacerbations during the follow-up period necessitating unplanned visits to health-care providers (OR 0.85; 95\% CI $0.54,1.34 ; 4$ trials; 981 participants), new exacerbations requiring additional oral steroids (OR 0.29. 95\% CI 0.10, $0.81 ; 1$ trial; 242 participants), and prevalence of vomiting (OR 3.05; CI 0.88, 10.55; 3 trials; 867 participants). Two of the four trials in the review reported comparable symptom scores between the two groups. Overall, these data suggest that dexamethasone has comparable (but not superior) efficacy and safety to prednisone. However, the trials had differences in terms of dose and/or duration of medications, outcomes studied, timing of outcome assessment, and methodological quality. Table I summarizes the characteristics of the trials and their differences.

One more trial comparing prednisone and dexamethasone has been recently published [10]. The trial characteristics are compared to the previous trials in 
JouRnAl CLUB

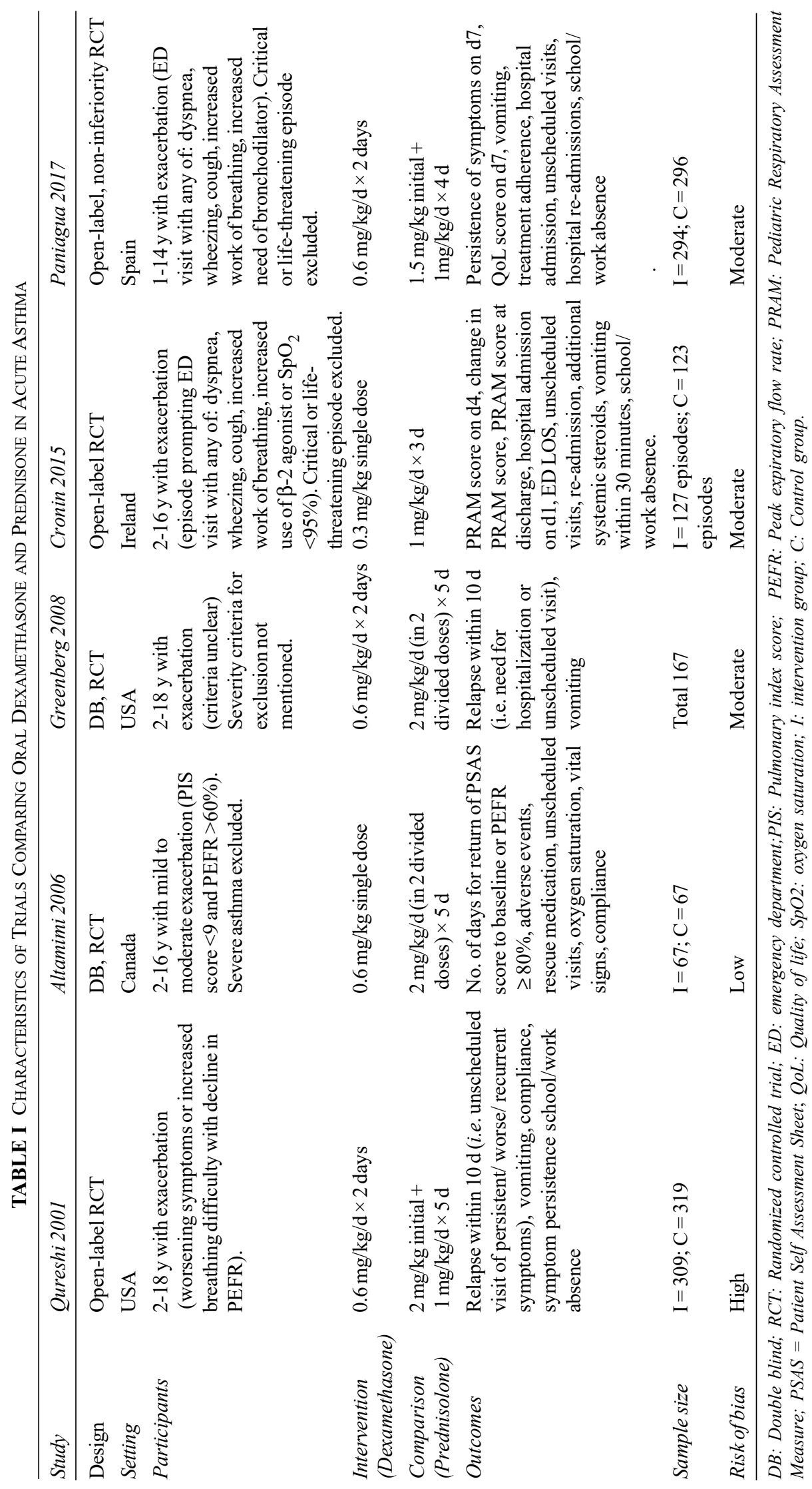

Indian Pediatrics

156

VOLUME 55-FeBRUARY 15, 2018 
Table I. The results showed that dexamethasone was comparable to prednisone with respect to persistence of symptoms (OR $0.93 ; 95 \%$ CI 0.67, 1.30), Quality of Life (QoL) score (mean difference 2.30 percentiles; 95\% CI $0.64,5.24$ ), admission to an observation unit (OR 1.07; $95 \%$ CI $0.71,1.63)$, hospital admission $(1.42 ; 95 \%$ CI 0.53 , 3.78 ), stay in the Emergency (mean difference 0.10 hours; 95\% CI -0.82, 1.02), unscheduled visits (OR 1.44; 95\% CI $0.60,3.42)$, hospital re-admission $(0.49 ; 95 \% \mathrm{CI} 0.04,5.43)$, vomiting $(0.48 ; 95 \% \mathrm{CI} 0.18,1.30)$, and use of additional steroids $(1.38 ; 95 \% \mathrm{CI} 0.71,2.69)$. Even the number of days of school/work missed were comparable. The only significant difference observed was that failure of adherence to treatment was lower with dexamethasone (OR $0.17 ; 95 \%$ CI $0.04,0.79$ ). The authors also reported a high degree of parental satisfaction on day 7 but this could not be compared (see below for details).

The additional data from this trial [10] facilitates an updated meta-analysis for major outcomes. This showed comparable results for initial hospital admission (OR 0.98; 95\% CI 0.69, 1.40; 4 trials; 1564 participants; $\mathrm{I}^{2}=0 \%$ ), hospital re-admission (OR 1.75; 95\% CI 0.67, 4.54; 4 trials; 1542 episodes; $\mathrm{I}^{2}=0 \%$ ), and unscheduled visits (OR 1.25 ; $95 \%$ CI $0.84,1.85 ; 5$ trials; 1538 participants; $\mathrm{I}^{2}=0 \%$ ). However, vomiting was significantly reduced with dexamethasone (OR 0.34; 95\% CI 0.19, 0.60; 4 trials; 1424 participants; $\mathrm{I}^{2}=28 \%$ ).

Critical appraisal: Methodological critical appraisal using the Cochrane Risk of Bias tool [11] is presented in Table II.

The trial included several methodological refinements. It was designed as a non-inferiority trial necessitating a larger sample size. In fact, this is the only non-inferiority trial comparing dexamethasone versus prednisone. The trial included fairly robust definitions/ criteria for asthma, exacerbations, and the scores used to calculate symptom persistence and quality of life. Although most outcomes were based on parental report, some of the data were retrieved from electronic records reducing the risk of bias in parental reporting.

However, there are some significant limitations worth mentioning. The investigators chose two patient-centric measures (viz symptom persistence and QoL score on day 7) as the primary outcomes rather than the conventional objective measures of improvement (such as symptom scores, need for admission, step-up of therapy/care, etc). The authors attempted to justify this decision, but the arguments are weak. Since three of the four previous trials were already available when this trial was started, it would have been better to align the outcomes to facilitate comparison with existing data. Further, both the primary outcomes were based on parental report, and that too, obtained over telephone, rather than a face-to-face interview. This makes it difficult to assess the reliability of these outcome measures.

Despite this, the investigators did not choose to ensure blinding of the parents/children and personnel collecting data over the phone. In a trial of this nature, this would have been relatively easy using the double-dummy design. The impact of absence of blinding of these groups is difficult to assess in this trial.

One of the major challenges in this trial is a very high proportion of children with persistence of symptoms at the end-point ( 7 days). In fact, $57 \%$ children who received dexamethasone and $58 \%$ of those receiving prednisone continued to be symptomatic well after therapy was discontinued. This suggests a high rate of treatment failure (even though it was comparable between the two groups). No clear explanation was offered by the authors for this; although the doses and durations in both groups

TABLE II CRITICAL APPRAISAL OF THE RCT

Participant baseline characteristics

Randomization

Allocation concealment

Blinding of participants and personnel

Blinding of outcome assessors

Incomplete outcome data

Selective outcome reporting

Other sources of bias

Overall assessment
The participants in both groups were comparable in terms of age, gender distribution, characteristics of the exacerbation, and symptom persistence.

Adequate; A computer program was used to generate the allocation sequence.

Adequate; The allocation of individual children was concealed in serially numbered opaque envelopes.

Inadequate; This was not done.

Inadequate; This was not done.

Unclear; The trial randomized 590 children, but despite planning an intention-to-treat analysis, results were reported only in 557. The proportion of children whose data were missing was $4.4 \%$ in dexamethasone group and $6.8 \%$ in prednisone group.

Adequate; All the outcomes planned, were measured and reported.

No obvious bias

Moderate risk of bias 
were in line with standard recommendations.

Further careful analysis of the primary outcome measures shows that the proportion of children with symptom persistence (in both groups) was higher at the end-point than at the baseline (56.6\% compared to $43.8 \%$ for dexamethasone; and $58.3 \%$ compared to $37.7 \%$ for prednisone). This seems strange, but no explanation has been offered. In fact, each of the components of the symptom persistence scoring system also showed worsening after therapy (than at baseline) in both groups. Further, the QoL score remained unchanged after therapy (80.0 vs 79.4 for dexamethasone; and 77.7 vs 79.5 for prednisone). This is also difficult to explain. It is possible that these apparently unusual observations could be related to data being obtained by direct interview at baseline, but by telephone at the end-point. This again highlights that the primary outcome measures in this study were not ideal.

In contrast, most of the secondary outcome measures did not suggest that the apparent treatment failure necessitated medical attention to the same extent. For example, there was very low hospitalization rate, hospital re-admission rate, unscheduled visits to healthcare provider(s), need for additional steroids, and school absenteeism. However, one outcome designated 'admission to observation unit' was present in nearly one-fifth of all children. Unfortunately, the details have not been described, but it could suggest need for further care in a significant proportion of the children (although the rate was comparable between the groups). These observations suggest that dexamethasone and prednisone had a very high (through comparable) lack of efficacy in this trial. The only outcome tilted in favor of dexamethasone was lack of adherence; although, it was extremely low in both groups. However, as this was also based purely on parental report, the veracity is questionable.

The investigators suggested very high parental satisfaction at the end-point; however, this outcome does not appear to have been measured in all the children. Strangely, parental satisfaction is reported in $99.3 \%$ and $96.0 \%$ in the dexamethasone and prednisone groups, respectively, whereas the respective absolute numbers are only 210 and 179 . This makes it difficult to interpret this outcome.

Last but not the least, the investigators planned intention-to-treat analysis. In its purest sense this implies that all randomized participants should appear in the analysis, and not only those for whom data are available, or those who complete the trial per protocol [12]. In this trial, 590 children were randomized (and should have constituted the denominator), whereas data were analyzed using only 557 children.

Extendibility: Both medications used in this trial are easily available in our setting in various formulations and packaging, making it easy to administer. Further, the possibility of reducing treatment duration from 5 days to 2 days (by using dexamethasone instead of prednisone) makes it an attractive proposition. However, the data from this trial do not provide compelling evidence to switch from the current standard of care to an alternative strategy. This is because there are several concerns with the internal validity of the trial (highlighted above). Further, the four previous trials also demonstrated only comparability, but not superiority of dexamethasone over prednisone.

Conclusion: This randomized trial showed that the treatment of acute asthma (except severe cases) with oral dexamethasone had comparable efficacy to oral prednisone, although a high rate of treatment failure was observed in both groups. Updated meta-analysis confirmed comparable efficacy outcomes, but vomiting was significantly lower with dexamethasone. This is a new finding not identified in the previous meta-analyses [9].

Funding: None; Competing interests: None stated.

JOSEPH L MATHEW Department of Pediatrics, PGIMER, Chandigarh, India. dr.joseph.l.mathew@gmail.com

\section{REFERENCES}

1. National Institute for Health and Care Excellence (NICE). Asthma Quality Standard 2013. Available from: https:// www.nice.org.uk/guidance/ng80/chapter/Recommendations \#initial-clinical-assessment. Accessed January 15, 2018.

2. Global Initiative for Asthma (GINA). Global Strategy for Asthma Management and Prevention. Available from: http:/ /ginasthma.org/2017-gina-report-global-strategy-forasthma-management-and-prevention/. Accessed January $15,2018$.

3. British Thoracic Society and Scottish Intercollegiate Guidelines Network. British Guideline on the Management of Asthma. Available from: https://www.britthoracic.org.uk/document-library/clinical-information/ asthma/btssign-asthma-guideline-2016/. Accessed January 15,2018

4. Arakawa H, Hamasaki Y, Kohno Y, Ebisawa M, Kondo N, Nishima S, et al. Japanese guidelines for childhood asthma 2017. Allergol Int. 2017;66:190-204.

5. Qureshi F, Zaritsky A, Poirier MP. Comparative efficacy of oral dexamethasone versus oral prednisone in acute pediatric asthma. J Pediatr. 2001;139:20-6.

6. Altamimi S, Robertson G, Jastaniah W, Davey A, Dehghani 
$\mathrm{N}$, Chen $\mathrm{R}$, et al. Single-dose oral dexamethasone in the emergency management of children with exacerbations of mild to moderate asthma. Pediatr Emerg Care. 2006;22:786-93.

7. Greenberg RA, Kerby G, Roosevelt GE. A comparison of oral dexamethasone with oral prednisone in pediatric asthma exacerbations treated in the emergency department. Clin Pediatr (Phila). 2008;47:817-23.

8. Cronin JJ, McCoy S, Kennedy U, An Fhailí SN,Wakai A, Hayden $\mathrm{J}$, et al. A randomized trial of single-dose oral dexamethasone versus multi dose prednisolone for acute exacerbations of asthma in children who attend the emergency department. Ann Emerg Med. 2016;67:593601.

9. Normansell R, Kew KM, Mansour G. Different oral corticosteroid regimens for acute asthma. Cochrane Database Syst Rev. 2016;5:CD011801.

10. Paniagua N, Lopez R, Muñoz N, Tames M, Mojica E, Arana-Arri E, et al. Randomized trial of dexamethasone versus prednisone for children with acute asthma exacerbations. J Pediatr. 2017;191:190-6

11. Cochrane Risk of Bias Tool (modified) for Quality Assessment of Randomized Controlled Trials. Available from:http://www.tc.umn.edu/ msrg/caseCATdoc/ rct.crit.pdf. Accessed November 20, 2017.

12. Cochrane Community. Glossary. Available from: http:// community.cochrane.org/glossary\#letter-I. Accessed January 15, 2018.

\section{Pediatric Pulmonologist's Viewpoint}

The investigators in this randomized, non-inferiority trial compared two doses of dexamethasone $(0.6 \mathrm{mg} / \mathrm{kg} / \mathrm{dose})$ with 5 day oral prednisone $(1-1.5 \mathrm{mg} / \mathrm{kg} /$ day $)$ in children 1 14 years of age with acute asthma presentation to emergency department (ED). At day 7, no difference between groups was noted for persistent symptoms, quality of life (QoL) score, admission rate, unscheduled ED return and vomiting. Adherence was greater in the dexamethasone group.

Previous studies that have explored this question have chosen to compare either single or two days of dexamethasone ( 0.3 or $0.6 \mathrm{mg} / \mathrm{kg} /$ dose) vs prednisone (1-2 $\mathrm{mg} / \mathrm{kg} /$ day) for 3 or 5 days. A meta-analysis in 2014, including six randomized controlled trials in children with acute asthma, comparing dexamethasone (oral or IM) and oral prednisone (5 days) indicated no difference in relative risk (RR) of relapse between the groups at any time point ( 5 days RR $0.90,95 \%$ CI $0.46,1.78 ; 10-14$ days RR 1.14, 95\% CI 0.77, 1.67). Dexamethasone group were less likely to experience vomiting in either the $\mathrm{ED}$ (RR 0.29, $95 \%$ CI $0.12,0.69)$ or home (RR $0.32,95 \%$ CI $0.14,0.74)[1]$.
International and Indian Academy of Pediatrics (IAP) asthma Guidelines are uniform in recommending systemic corticosteroids for asthma exacerbations, except the mildest severity, for speedy resolution and prevention of relapse and hospital admission. Traditionally oral prednisone/prednisolone has been the most commonly recommended corticosteroid. BTS-SIGN 2016 guidance recommends oral prednisolone for 3 days, or IV hydrocortisone when unable to tolerate oral medication [2]. GINA 2017 guideline recommends either oral prednisolone for 3-5 days or oral dexamethasone for 2 days [3]. Dexamethasone is associated with metabolic adverse effects if continued beyond 2 days.

Oral dexamethasone has the advantage of longer biological half life compared to prednisone (36-72 h vs $12-36 \mathrm{~h}$ ), good bioavailability, and better palatability. Oral prednisone is bitter in taste and patients report vomiting leading to poor adherence. Dexamethasone is more palatable and the two day course ensures better compliance. Cost-effective analysis model indicates 2 days dexamethasone compared to 5 days prednisone was cost-saving on both direct and indirect measures(missed parental work days, parental salary) based on US and Canadian cost estimates [4].

These comparisons and recent studies suggest that a 2-day dexamethasone course can be explored as an alternative option to the longer prednisolone regimen in management of acute asthma in children.

Funding: None; Competing interests: None stated.

Mandeep Kaur Walia

Clinical Fellow, Respiratory Division, BC Children's Hospital, University of British Columbia, Canada. mkwalia2000@yahoo.co.in

\section{REFERENCES}

1. Randolph C. Dexamethasone for acute asthma exacerbations in children: A meta-analysis. Pediatrics. 2014;134 (Suppl 3):S178-9.

2. BTS/SIGN British Guideline for the management of asthma, 2016, SIGN 153. Available from: http:// www.sign.ac.uk/sign-153-british-guideline-on-themanagement-of-asthma.Accessed January 10, 2018.

3. Global Initiative for Asthma, GINA, 2017. Available from: http://ginasthma.org/2017-gina-report-globalstrategy-forasthma-management-and-prevention. Accessed January 10, 2018.

4. Andrews AL, Wong KA, Heine D, Scott Russell W. A costeffectiveness analysis of dexamethasone versus prednisone in pediatric acute asthma exacerbations. Acad Emerg Med. 2012;19:943-8. 